EPJ Web of Conferences 70, 00018 (2014)

DOI: 10.1051/epjconf/20147000018

(C) Owned by the authors, published by EDP Sciences, 2014

\title{
Thermal characteristics confronting trace anomaly and intrinsic canonical structure of QCD
}

\section{A traceback from hypotheses of mainly second order phase transition with respect to energy density in QCD}

\author{
Peter Minkowski ${ }^{1}$ and Sonia Kabana ${ }^{2}$ \\ ${ }^{1}$ Albert Einstein Center for Fundamental Physics - ITP, University of Bern, Switzerland \\ ${ }^{2}$ University of Nantes and Laboratory SUBATECH, Nantes, France
}

\begin{abstract}
Based on the hypotheses assumed but left without proof in the paper [1-2010] the structural ingredients implementing these proofs are taken up (sketched), as they relate to the gauge- and boundary-conditions obeyed by thermal parameters and rooted in the infrared completion of the theory.
\end{abstract}

\section{Characterization of Bose condensates}

As entry point let me present three thermal systems with superfluid-fluid phase transition on the one hand and a superconducting one (type I or II) on the other, but all three related to (here thermal) Bose condensates. The three systems possess minimally two intensive thermal variables, e.g. temperature $\mathrm{T}$ and density or molar density $\varrho_{n}=N / V$, the latter equivalent to a chemical potential for conserved baryonic- and electronic- charge respectively.

1) He-4 single atom He-4 II superfluid - He-4 I normal fluid transition

The Bose condensing field is a single He-4 boson with an extensive fraction of atoms all sharing the same wave function, illustrated in Fig. 1.

$$
T_{c} \equiv T_{c r}=O\left(1^{\circ} \mathrm{K}\right)
$$

2) He-3 fermion-pair-condensed superfluid - normal fluid transition

The Bose condensing field is made up of a pair of fermionic He-3 atoms, illustrated in Fig. 2.

$$
T_{c} \equiv T_{c r}=O\left(10^{-3 \circ} \mathrm{K}\right)
$$

3) electron-pair-condensed superconducting - normal conducting material (e.g. Niobium)

The Bose condensing field is made up of a pair of fermionic electrons, illustrated in Fig. 3.

$$
T_{c} \equiv T_{c r}=O\left(2-100^{\circ} \mathrm{K}\right)
$$

A short list of historical references is included in the reference section on the page marked $\mathrm{rH}-1$.

A few remarks on the 3 systems chosen to exemplify Bose condensates are in order here.

This is an Open Access article distributed under the terms of the Creative Commons Attribution License 2.0, which permits unrestricted use, distribution, and reproduction in any medium, provided the original work is properly cited. 
EPJ Web of Conferences

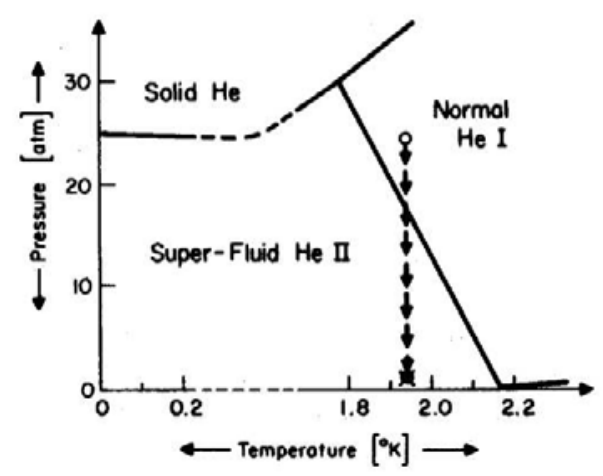

Figure 1. He-4 II superfluid - He-4 I fluid phase boundary from ref. [2-1993]

\section{The superfluid phases of ${ }^{3} \mathrm{He}$}

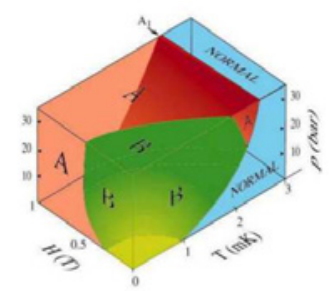

Figure 2. He-3 A,B superfluid - He-3 fluid phase boundaries from ref. [3-2011]

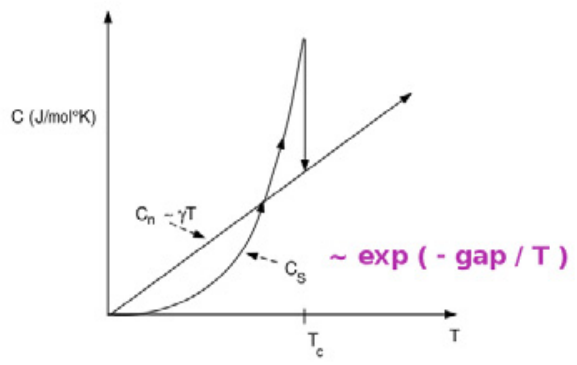

Figure 3. Specific heat in the superconducting phase $\longleftrightarrow$ 'no latent heat, indicates that the transition is continuous or second order', from ref. [4-2001],(Bardeen, Cooper and Schrieffer) 
1) Lev Davidovich Landau did work on the quantum nature of first the He-4 Bose-liquid 1936-38, as a member of the Institute directed by Pyotr Leonidovich Kapitsa, as well as later 1956-58, on Fermi-liquids including superfluid He-3.

In the theoretical part of this endeavour the rooting in elementary field theory was not fully achieved, but the prediction of second sound with velocity distinct from primary sound waves was subsequently proven correct experimentally.

2) The discovery of superconductivity by Kammerling Onnes recently celebrated its centenary. Yet the 'Microscopic Theory of Superconductivity' by B-C-S in 1957 raised - rightly so - much general attention. It infers fermion-pairing of two electrons and condensation of these (Cooper-) pairs also leaving the field theoretic roots incomplete. An important role towards the goal of field quanta and Bose-condensation was derived by Nikolai Nikolaevich Bogoliubov [H3-1947].

3) In the ensuing discussion in QCD we shall conceive this theory completely switching off electromagnetism and all associated electrically charged and neutral leptons. While this is a clear simplification, associations with most commonly known thermal phases is at the same time accordingly restricted as well as dangereous.

\subsection{Bose condensates and trace anomaly in QCD}

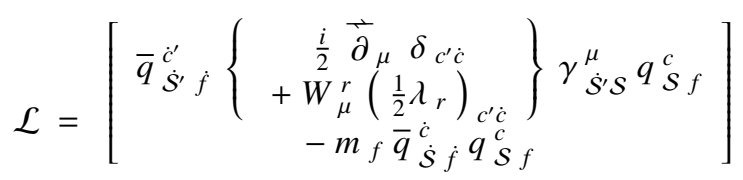

$$
\begin{aligned}
& -\frac{1}{4 g^{2}} B^{\mu v r} B_{\mu \nu}^{r}+\Delta \mathcal{L} \\
& W_{\mu}^{r} \equiv-v_{\mu}^{r} \text { for identification of convention for potentials } \\
& \text { quarks : } c^{\prime} c=1,2,3 \text { color } f=1, \cdots, 6 \text { flavor } \\
& \mathcal{S}^{\prime}, \mathcal{S}=1, \cdots, 4 \text { spin } m_{f} \text { mass } \\
& \text { gauge bosons : } \mathcal{L}_{B}=-\frac{1}{4 g^{2}} B^{\mu \nu r} B_{\mu \nu}^{r} \\
& B_{\mu \nu}^{r}=\partial_{\mu} W_{v}^{r}-\partial_{\nu} W_{\mu}^{r}+f_{r s t} W_{\mu}^{s} W_{\nu}^{t} \longleftarrow\left(W_{\mu}^{r} \equiv-v_{\mu}^{r}\right) \\
& r, s, t=1, \cdots, \operatorname{dim}\left(G=S U 3_{c}\right)=8 \\
& \text { Lie algebra labels, }\left[\frac{1}{2} \lambda^{r} \frac{1}{2} \lambda^{s}\right]=i f_{r s t} \frac{1}{2} \lambda^{t} \\
& \text { perturbative rescaling : } \\
& W_{\mu}^{r}=g W_{\mu \text { pert }}^{r} B_{\mu \nu}^{r}=g B_{\mu \nu \text { pert }}^{r}
\end{aligned}
$$

Completing $\Delta \mathcal{L}$ in Fermi gauges

$$
\begin{aligned}
& \Delta \mathcal{L}=\left\{\begin{array}{c}
-\frac{1}{2 \eta g^{2}}\left(\partial_{\mu} W^{\mu r}\right)^{2} \\
+\partial^{\mu} \bar{c}^{r}\left(D_{\mu} c\right)^{r}
\end{array}\right\} ; \eta: \text { gauge parameter } \\
& \text { ghost fermion fields }: c, \bar{c} ;\left(D_{\mu} c\right)^{r}=\partial_{\mu} c^{r}+f_{r s t} W_{\mu}^{s} c^{t} \\
& \text { gauge fixing constraint : } C^{r}=\partial_{\mu} W^{\mu r}
\end{aligned}
$$




$$
\begin{aligned}
& \left\{\vartheta_{\mu}^{\mu} \quad=\sum_{f} m_{f} S_{\dot{f} f}+\delta_{0}\right\}(x) \\
& \left\{\partial^{\mu}\left(j_{\mu}^{5}\right)^{S}=2\langle m\rangle i P^{S}+\delta_{5}\right\}(x) \\
& \delta_{0}=-\left(-2 \beta(g) / g^{3}\right)\left[\frac{1}{4}(:) B_{\mu v}^{t} B^{\mu v t}(:)\right]_{\rightarrow \text { ren.gr.inv }} \\
& \delta_{5}=\left(2 N_{f l}\right) \frac{1}{8 \pi^{2}}\left[\frac{1}{4}(:) B_{\mu \nu}^{t} \widetilde{B}^{\mu v t}(:)\right]_{\rightarrow \text { ren.gr.inv }} \\
& -\beta / g^{3}=\frac{1}{16 \pi^{2}} b_{0}+O(X) ; X=g^{2} /\left(16 \pi^{2}\right. \\
& \underbrace{\text { the two central anomalies in a nutshell }} \\
& \left\{\vartheta_{\mu}^{\mu} \quad=\sum_{f} m_{f} S_{\dot{f} f}+\delta_{0}\right\}(x) \\
& \left\{\partial^{\mu}\left(j_{\mu}^{5}\right)^{S}=2\langle m\rangle i P^{S}+\delta_{5}\right\}(x) \\
& \delta_{0}=-\left(-2 \beta(g) / g^{3}\right)\left[\frac{1}{4}(:) B_{\mu v}^{t} B^{\mu v t}(:)\right]_{\rightarrow \text { ren.gr.inv }} \\
& \delta_{5}=\left(2 N_{f l}\right) \frac{1}{8 \pi^{2}}\left[\frac{1}{4}(:) B_{\mu v}^{t} \widetilde{B}^{\mu v t}(:)\right]_{\rightarrow \text { ren.gr.inv }}
\end{aligned}
$$

\section{What is what in the expressions for the central anomalies in eq. 8 above}

1) $\vartheta_{\mu \nu}(x)=\vartheta_{\nu \mu}(x)$ with $\partial^{\mu} \vartheta_{\mu \nu}=0$ denotes the conserved, symmetric and local $S U 3_{c}$ gauge invariant energy momentum density tensor.

2) $\left(j_{\mu}^{5}\right)^{S}(x)$ stands for the flavor singlet axial current $\sum_{f}(:) \bar{q}_{f}^{\dot{c}} \gamma_{\mu} \gamma_{5} q_{f}^{c}(:)(x)$.

3) The flavor diagonal scalar and pseudoscalar densities are denoted

$S_{\dot{f} f}(x)=(:) \bar{q}_{\dot{f}}^{\dot{c}} q_{f}^{c}(:)(x), P_{\dot{f} f}(x)=(:) \bar{q}_{\dot{f}}^{\dot{c}} \gamma_{5} q_{f}^{c}(:)(x)$ (no sum on $\mathrm{f}$ ).

4) $S_{\dot{f} f}$ are hermitian, $P_{\dot{f} f}$ antihermitian local densities, with $\left(j_{\mu}^{5}\right)^{S} P^{S}$ implying a projection on the flavor singlet contributions. $m_{f}$ denotes the nonnegativ quark mass pertaining to flavor $\mathrm{f}$.

5) The symbols (:) (:) denote normal ordering, which in the restriction to perturbative approximations can be thought of as normal ordering with respect to noninteracting massless (or massive) quark fields and noninteracting massless gauge bosons.

In contradistinction to quark fields, whose infrared divergences are cured by any finite quark mass terms, even if only used as regulators, the gauge boson fields always retain in the perturbative approximation their infrared divergences. 


\section{Consequential condensate categories}

1) In QCD without QED and leptons there are no Bose condensates relative to base fields, both fermionic violating CPT as well as bosonic. The exclusion is a consequence of strong boundary conditions maintaining unbroken local gauge invariance barring the color octet gauge bosons from forming a condensate.

2) Bose condensates thus involve composite fields, minimally quark-antiquark color neutral pairs, but possibly also higher color neutral even multiples of quarks and antiquarks for composite fermion fields, as well as again minimally pairs of gauge bosons, but possibly also color neutral arbitrary even and odd multiples thereof.

E.g. pair field substrates with quantum numbers $J^{P C}=0^{++}$are shown in eq. 9 below

\begin{tabular}{|c|c|}
\hline fermion pairs & gauge boson pairs \\
\hline$(:) \bar{q}_{f}^{\dot{c}} q_{f}^{c}(:)(x)$ & $\frac{1}{4}(:) B_{\mu \nu}^{t} B^{\mu v t}(:)(x)$ \\
\hline
\end{tabular}

\section{The dominantly second order phase transition for vanishing chemical potentials}

In this section and second part the hypothesis is investigated, that the thermal structure of QCD phases at and near zero chemical potentials is determined by long range coherence, inducing the gauge boson pair condensate, and its thermal extension, representing a fundamental order parameter.

This work relates to reference [1-2010].

A consistent framework for thermal behaviour including interactions is derived in which the condensate does not produce any latent heat as it vanishes at the critical temperature inducing a second order phase transition with respect to energy density, neglecting eventual numerically small critical exponents. We mention the extreme situation of a solvable model in 2 dimensions, exhibiting an infinite order phase transition [5-1973].

The aim was to tackle the questions left open in our previous work [6-2001] and to illustrate the effects representing the (dominantly) second order transition with respect to energy density - in principle not in numerical detail - as they affect the other thermodynamic variables like pressure and velocity of sound.

Localization and delocalization of color fields are thus separated by a unique critical temperature. The existence and nature of the QCD phase transition is theoretically amenable to a universal description underlying a thermal system with all its general and specific restrictions, but is tantamount to a full completion of the infrared instability of the theory.

Lattice QCD calculations lead to a very clear picture, establishing the lack of any phase transition at zero chemical potentials [7-2010, 8-2009].

\subsection{Energy momentum density tensor and vacuum condensates : gauge boson - and $q \bar{q}$ - pairs}

We recall the local, symmetric gauge invariant and conserved nature of the energy momentum density tensor $\vartheta_{\mu \nu}$, as displayed in eqs. 7 and 8 as well as the form of the trace anomaly (eq. 9). The above properties imply exact Poincaré invariance. 


$$
\begin{aligned}
& \left\{\vartheta_{\mu v}=\vartheta_{v \mu}\right\}(x) ; \partial^{v} \vartheta_{\mu v}=0 \\
& \vartheta_{\mu}^{\mu}=\sum_{f} m_{f} S_{\dot{f f}}(x)+\delta_{0}(x) \\
& \delta_{0}(x)=-\frac{b_{0}}{8 \pi^{2}}\left[\frac{1}{4}(:) B_{\mu \nu}^{t} B^{\mu v t}(:)(x)\right]_{\text {ren.gr.inv. }} \\
& b_{0}=11-\frac{2}{3} N_{f l}
\end{aligned}
$$

In eq. $10 \frac{1}{4}(:) B_{\mu \nu}^{t} B^{t \mu \nu}(:)(x)$ denotes the normal ordered local gauge boson (field strength-) bilinear, and $S_{\dot{f f}}(x)=(:) \bar{q}_{\dot{f}}^{\dot{c}} q_{f}^{c}(:)(x)$ the scalar $q-\bar{q}$ bilinear pertaining to flavor $\mathrm{f}$, introduced in eq. 9 in section 1.

We thus consider vacuum expected values of the local operators in eq. 10, which by translation invariance are independent of position $x$, suppressed in the following

$$
\begin{aligned}
\eta^{\mu v}\left\langle\Omega\left|\vartheta_{\mu \nu}(x)\right| \Omega\right\rangle= & -\frac{b_{0}}{8 \pi^{2}}\left\langle\Omega\left|\frac{1}{4}(:) B_{\mu \nu}^{t} B^{t \mu v}(:)(x)\right| \Omega\right\rangle \\
& +m_{f}\left\langle\Omega\left|S_{f f}(x)\right| \Omega\right\rangle
\end{aligned}
$$

$\langle\Omega|$ (:) $\frac{1}{4} B_{\mu \nu}^{t} B^{t \mu \nu}(:)|\Omega\rangle=\mathcal{B}^{2}$ shall be called the gauge boson pair vacuum condensate, abbreviated by $\mathcal{B}^{2}$.

$\left\langle\Omega\left|S_{\dot{f} f}\right| \Omega\right\rangle \neq 0$ induces spontaneous chiral symmetry breaking and is generally called $q-\bar{q}$ vacuum condensate.

In connection with normal ordering ambiguities it is important to admit in the precise form of the energy momentum tensor a nontrivial vacuum expected value, which as a consequence of exact Poincaré invariance must be of the form

$$
\begin{aligned}
& \left\langle\Omega\left|\vartheta_{\mu \nu}(x)\right| \Omega\right\rangle=-\eta_{\mu \nu} p_{v a c} \\
& \left\{\eta_{\mu \nu}=\operatorname{diag}(1-1-1-1) ; p_{v a c}=-\rho_{v a c}\right\} \\
& \text { independent of } x \rightarrow \\
& \Delta \vartheta_{\mu \nu}(x)=\vartheta_{\mu \nu}(x)-\left\langle\Omega\left|\vartheta_{\mu \nu}(x)\right| \Omega\right\rangle|\Omega\rangle\langle\Omega| \\
& \text { with } \partial^{v} \Delta \vartheta_{\mu \nu}(x)=0 ;\left\langle\Omega\left|\Delta \vartheta_{\mu \nu}(x)\right| \Omega\right\rangle=0
\end{aligned}
$$

In eq. $12 P_{\Omega}=|\Omega\rangle\langle\Omega|$ denotes the projector on the ground state.

From the two local, conserved tensors in eq. 12 only $\Delta \vartheta_{\mu \nu}(x)$ with vanishing vacuum expected value is acceptable as representing the conserved 4 momentum operators and their densities yielding the integral form

$$
\widehat{P}_{\mu}=\int{ }_{t} d^{3} x \Delta \vartheta_{\mu 0}(t, \vec{x})
$$

We use here throughout strictly thermal, 'extension in phase space' associated potentials, depending in subtle ways on vacuum condensates. To these potentials no vacuum associated spontaneous parameters like $p_{v a c}=-\rho_{v a c}$, defined in eq. 12 , contribute in a direct way, dominating in the limit $T \rightarrow 0 ; \mu_{\alpha} \equiv 0$.

From eqs. 11 and 12 we obtain the relation and estimates. Errors may be underestimated. 


$$
\begin{aligned}
p_{\text {vac }} & =\frac{9}{32 \pi^{2}} \mathcal{B}^{2}+\frac{1}{4} \widetilde{\Lambda}=\left\{\begin{array}{l}
0.00302 \mathrm{GeV}^{4} \\
0.00658 \mathrm{GeV}^{4}
\end{array}\right. \\
\mathcal{B}^{2} & =\left\{\begin{array}{l}
0.125 \mathrm{GeV}^{4}[9-1979] \\
0.250 \mathrm{GeV}^{4}[10-1998]
\end{array}\right. \\
\widetilde{\Lambda} & =-\sum_{f} m_{f}\left\langle\Omega\left|S_{f f}\right| \Omega\right\rangle \\
& \sim f_{\pi}^{2}\left(\frac{1}{2} m_{\pi}^{2}+m_{K}^{2}\right)=0.00217 \mathrm{GeV}^{4}
\end{aligned}
$$

\subsection{Construction of a thermal model including interactions}

The central defining quantities, characterizing existence and order of the phase transition, are - for zero chemical potentials - pressure, energy density and entropy density.

We follow the strategy layed out in ref. [6-2001] taking into account the modifications described above, distinguishing two eventual phases

1) the hadronic (hg)-phase, with color localized within stable hadrons and selected hadron resonances. Thermal potentials of the (hg)-phase are approximated by those of free hadrons, neglecting decay widths, as described in ref. [6-2001].

2) the quark-antiquark-gauge boson (qg)-phase, wherein thermal potentials are related but not equal to those of free quarks and antiquarks, restricted to the flavors u,d,s and c, and 8 gauge bosons pertaining to the gauge group $S U 3_{c}$. Next we describe the modeling of interactions in the (qg)-phase, which deviates from noninteracting constituents assumed in ref. [6-2001].

We introduce for the (qg)-phase, the Gibbs density $g_{q g}^{(0)}$ and energy density $\varrho_{e q g}^{(0)}$, where the superscript ${ }^{(0)}$ refers to free tricolored quark-, antiquark- flavors u, d, s, c and eightfold colored gauge bosons

$$
\begin{aligned}
& g_{q g}^{(0)}(T)=\sum_{\alpha_{q g}} w_{\alpha_{q g}}\left(1 /\left(2 \pi^{2}\right)\right) \int_{m_{\alpha_{q} q}}^{\infty} l E p d E \\
& w_{\alpha_{q g}}=\left(2 \operatorname{spin}_{\alpha_{q g}}+1\right)\left\{\begin{array}{l}
3 \text { for } q, \bar{q} \\
8 \text { for } g
\end{array}=\left\{\begin{array}{c}
6 \text { for } q, \bar{q} \\
16 \text { for } g
\end{array}\right.\right. \\
& \beta \equiv 1 / T ; l=\mp \log [1 \mp \exp (-\beta E)] \\
& \varrho_{e q g}^{(0)}(T)=-(d / d \beta) g_{q g}^{(0)}(T)=T^{2}(d / d T) g_{q g}^{(0)}(T)
\end{aligned}
$$

In eq. 15 the index $\alpha_{q g}$ runs over the different constituents of the (qg) phase, while $w_{\alpha_{q g}}$ denotes the multiplicity beyond momentum phase space associated with the constituent $\alpha_{q g}$. The sign ( $\mp$ ) in the expression for $l$ is - for bosons and + for fermions.

We choose the following masses for quark flavors $\mathrm{u}, \mathrm{d}, \mathrm{s}, \mathrm{c}$

$$
\left[m_{u}=0.00525, m_{d}=0.00875, m_{s}=0.175, m_{c}=1.27\right] \mathrm{GeV}
$$

The masses of the $\mathrm{u}, \mathrm{d}, \mathrm{s}$ light flavors as well as their ratios $m_{u}: m_{d}: m_{s}=3: 5: 100$, within generous ranges of $\pm 20 \%$, should not play any decisive role here.

The gauge boson pair vacuum condensate is thought to be the cause of the stable embedding of a wide range of (light) quark masses, without wrecking approximate flavor symmetries. 
The inclusion of the charmed quark serves the purpose to check whether it has any significant influence on the thermal parameters in the region of $T_{c} \sim 0.2 \mathrm{GeV}$, wich turns out to be in the few percent range.

We proceed to modify the free quark antiquark gauge boson (qg-) parametrization of the Gibbs potential and the energy density, which for $\mu_{\alpha}=0$ must obey the exact relation

$$
\begin{aligned}
& g_{q g}=g_{q g}\left(T \equiv \beta^{-1}\right) ;-(d / d \beta) g_{q g}(T)=\varrho_{e q g}(T) \\
& \text { and } g_{q g} \leftrightarrow g_{h g}, \varrho_{e q g} \leftrightarrow \varrho_{e h g} ; h g: \text { hadron gas }
\end{aligned}
$$

The Gibbs- and energy-densities in the hadron phase are constructed from the expressions analogous to the ones given in eq. 15, where the index $\alpha_{q g} \rightarrow \alpha_{h g}$ runs over a suitable choice of hadrons and hadron resonances as defined in ref. [6-2001] with real masses and again neglecting interactions among these states. The ensuing parametrization of interactions is understood as representing the phase structure in principle and not in numerical detail. The modeling of interactions in the qg-phase is performed setting two parameters $k \Delta g$, independent of temperature, as approximately parametrizing the interaction in the assumed phase called qg - in a limited region of $T \geq T_{c r} \equiv T_{c}$

$$
\begin{array}{ll}
\varrho_{e q g}(T ; k) & =k \varrho_{e q g}^{(0)}(T) \\
g_{q g}(T ; k, \Delta g) & =k g_{q g}^{(0)}(T)-\Delta g
\end{array}
$$

The parameter $0<k<1$ is taking into account the reduction of Gibbs density or pressure

relative to the noninteracting (Stefan-Boltzmann) limit, noted in perturbative QCD calculations of thermal parameters for $T \simeq T_{c}$ of interest here [11-2007], while the second parameter $\Delta g$ is an integration constant from the differential equation (eq. 17), which is satisfied for any values of $(k \Delta g)$.

We proceed in two steps to map out the structure of the phase transition, using $T_{c} \equiv T_{c r}$

I : the condition determining $T_{c} \leftrightarrow k$

The equality of the energy densities - in the hadron phase $\varrho_{e}$ had for $T \leq T_{c}$ as outlined in ref. [6-2001] and in the qg-phase as defined in eq. $18 \varrho_{e q g}$ for $T \geq T_{c}$ determine the critical temperature

$$
\varrho_{e \text { had }}(T)=\varrho_{e q g}(T ; k) \leftrightarrow T=T_{c}(k)
$$

The matching (eq. 19) is further restricted to yield the value

$$
T_{c} \sim 0.2 \mathrm{GeV} \leftrightarrow k \sim 0.452 ; \text { for Ntype }=65
$$

in accordance with the estimate of one of us [12-1988].

II : the condition avoiding singular behaviour of pressure gradient

This condition implies

$$
g_{h g}\left(T_{c}\right)=g_{q g}\left(T_{c} ; k \Delta g\right) \leftrightarrow \Delta g=\Delta g\left(T_{c}(k)\right)
$$

and determines $\Delta g$

$$
\Delta g \sim 0.00753 \mathrm{GeV}^{3} \simeq 0.94 \mathrm{fm}^{-3} \text { for Ntype }=65
$$




\subsection{Results and discussion}

We present figures 4 - 10 in sequence, each followed by an extended caption.

Figure 4 shows how the transition temperature is determined and its stable variation, for the two sets of resonances, Ntype 65 and Ntype 26 forming the HRG $\leftrightarrow$ hg. The two sets are described in chapter 2, tables 1 - 8 and in Appendix 1, tables 9 - 11 and figures 8 - 10 .

Figure 5 shows in detail the energy- and Gibbs densities for the selected choice Ntype 65, as well as on the parameters $k \Delta g$ as defined in eqs. 17 - 18 in subsection 2.2.

Figure 6 shows the second order nature of the transition - unmodified and modified on the quarkgluon-side by subleading critical exponents - of the quantity $\varrho_{e}(T) / T^{4}$, piecewise described by $\varrho_{e h g(-65)} / T^{4}$ for $T \leq T_{c}$ from the HRG side and $\rho_{e \text { qg no mod (mod) }} / T^{4}$ for $T \geq T_{c}$ from the quark-gluon side, as defined in subsection 2.2.

Figure 7 shows the third order nature of the transition under the same conditions as underlying figure 3 for the quantity $p / T^{4}$, piecewise described by $p_{e} h(-65) / T^{4}$ for $T \leq T_{c}$ from the HRG side and $p_{\text {qg no mod }(\bmod )} / T^{4}$ for $T \geq T_{c}$ from the quark-gluon side.

Figure 8 shows the second order nature of the transition under the same conditions as figures 3 and 4 for the quantity dscale $=\left(\varrho_{e}-3 p\right) / T^{4}$, displaying the form of an 'indian tent'. The transition is piecewise described by dscale ${ }_{h g}$ for $T \leq T_{c}$ from the HRG side and dscale $q g$ no mod (mod) for $T \geq T_{c}$ from the quark-gluon side.

The modification by subleading critical exponents is given in eq. 23 below.

Figure 9 shows the quantities forming the 'indian tent' identical to figure 5. The quantity dscale obtained in ref. [7-2010] from lattice simulation of QCD under the same thermal conditions is also plotted for comparison.

Figure 10 shows the first order nature of the transition of the square of the velocity of sound, under the same conditions as for figures $6-9$, piecewise described by the quantities $v_{h g}^{2}$ for $T \leq T_{c}$ from the HRG side and $v_{q g \text { no mod }}^{2}$ for $T \geq T_{c}$ from the quark-gluon side. Only the unmodified setting is used for $v_{g q}^{2}$.

In figures 6 - 9 the modifications of $\varrho_{e} q g$ mod $/ T^{4}$ and $p_{q g}$ mod $/ T^{4}$ introduce a subleading critical exponent $v$ in the vicinity of $T=T_{c}$ allowing the free quark-gluon limits to be reached for $T \rightarrow \infty$. The modified quantities are defined as

$$
\begin{aligned}
& \left\{\begin{array}{c}
\varrho_{e}^{v}{ }_{q g}^{v} \\
p_{q g}^{v}
\end{array}\right\}=\left\{\begin{array}{l}
f \bmod \left(v T / T_{c}\right) \varrho_{e q g}+f \bmod 1\left(v T / T_{c}\right) p_{q g} \\
f \bmod \left(v T / T_{c}\right) p_{q g}
\end{array}\right\} \\
& \operatorname{fmod}\left(v T / T_{c}\right)=1+\left|1-T_{c} / T\right|^{2 v}(1 / k-1) \\
& \operatorname{fmod} 1\left(v T / T_{c}\right)=T(d / d T) \operatorname{fmod}\left(v T / T_{c}\right)
\end{aligned}
$$

The relations in eq. 23 ensure that the differential equation in eqs. 24 and 17 is satisfied.

$$
\varrho_{e}(T)=-\partial_{\beta} g(T), g(T)=\beta p(T) ; s(T)=\partial_{T} p(T) ; \beta=T^{-1}
$$

To Fig. 4 : In units of $\mathrm{GeV}^{4}$ and $\mathrm{GeV}^{3}$ for energy- and gibbs densities the same ordinates are used. The upper offset ordinate corresponds to Ntype $=65$ and is marked by ticmarks in red, while the lower ordinate corresponds to Ntype 26, with ticmarks in black. 


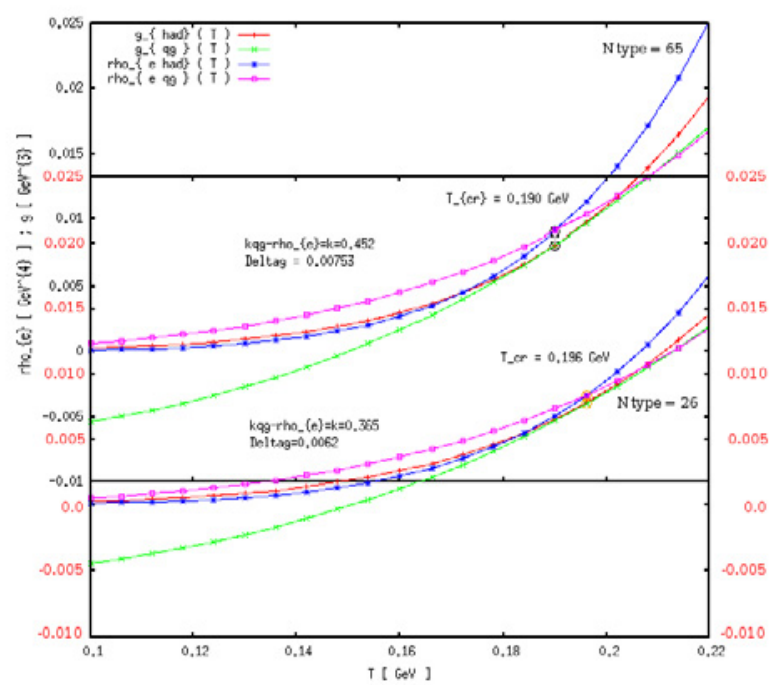

Figure 4. Dependence of energy- and Gibbs densities $\left(\varrho_{e} g\right)_{h g}$ pertaining to a free hadron gas,for two, offset, choices of spectra: Ntype $=65 \supset 26$, and associated $\left(\varrho_{e} g\right)_{q g}$ no mod .

The two conditions for a second, third order transition with respect to $\varrho_{e} g$

$$
\left\{\begin{array}{c}
\varrho_{e} \\
g
\end{array}\right\}_{h g}(T)=\left\{\begin{array}{c}
\varrho_{e} \\
g
\end{array}\right\}_{q g}(T) \text { for } T=T_{c}
$$

are marked by two $\circ$ symbols separately for Ntype $=65,26$. The two sets of parameters $T_{c} k(\equiv$ $r k) \Delta g$ given in eqs. 21,22 become

\begin{tabular}{cccc}
$T_{c}$ & $k$ & $\Delta g$ & Ntype \\
\hline $0.190 \mathrm{GeV}$ & 0.452 & $0.00753 \mathrm{GeV}^{3}$ & 65 \\
$0.196 \mathrm{GeV}$ & 0.365 & $0.0062 \mathrm{GeV}^{3}$ & 26
\end{tabular}

The quantities with suffix ${ }_{q g}$ refer to unmodified ones (no mod). 


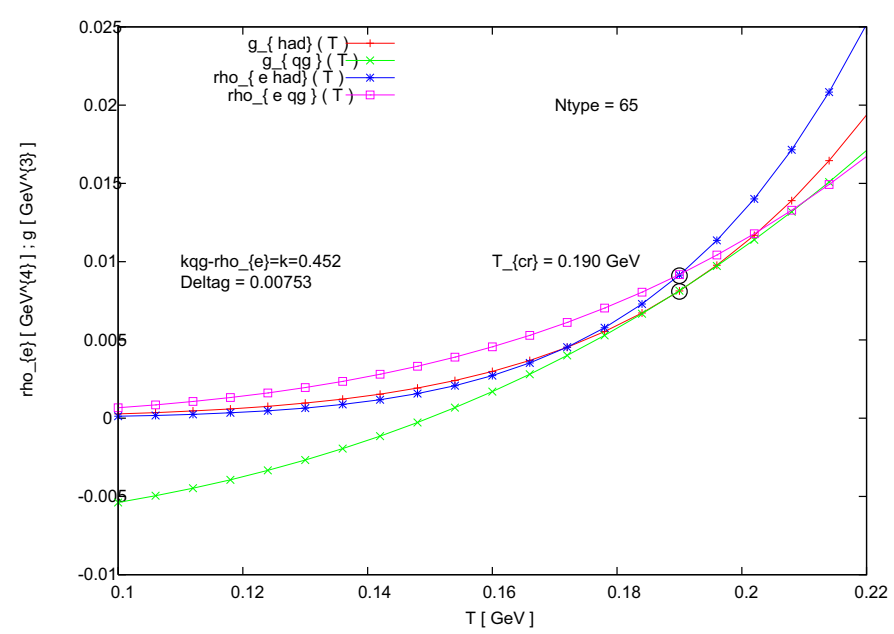

Figure 5. The upper offset case in Fig. 4 for Ntype $=65$ is displayed separately. Both captions direct and extended to Fig. 4 also refer to Fig. 5. Ntype $=65$ is subject of more detailed study in the following.

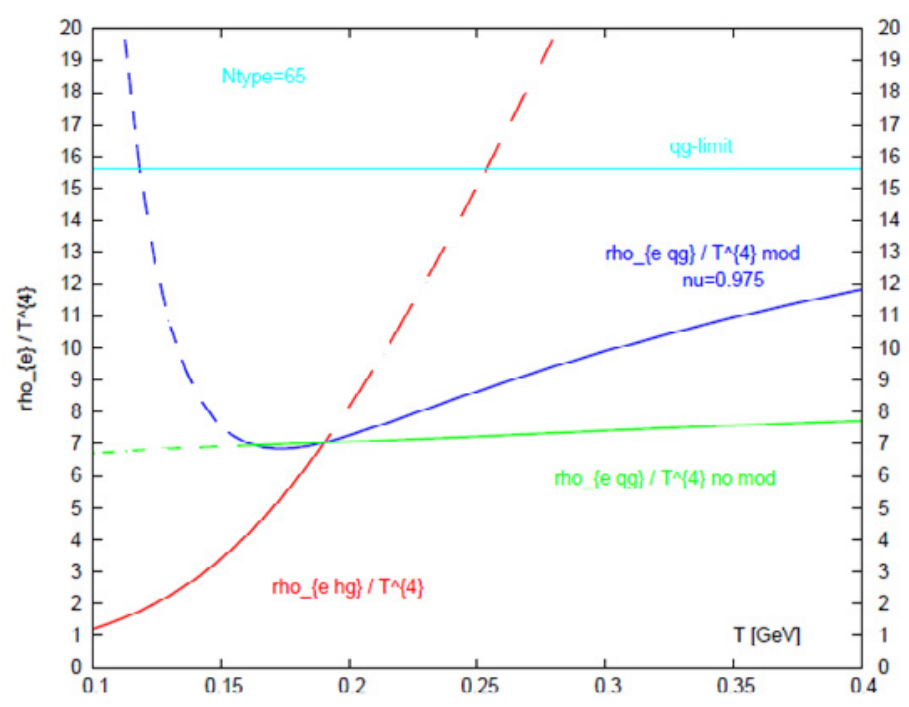

Figure 6. The enumeration in subsection 2.3 with respect to Figure 6 is repeated below.

It shows the second order nature of the transition - unmodified and modified on the quark-gluonside by subleading critical exponents - of the quantity $\varrho_{e}(T) / T^{4}$, piecewise described by $\varrho_{e h g(-65)} / T^{4}$ for $T \leq T_{c}$ from the HRG side and $\rho_{e \text { qg no mod (mod) }} / T^{4}$ for $T \geq T_{c}$ from the quark-gluon side, as defined in section 2 and subsection 2.2. The three curves are represented with dashed lines outside their range of validity. The quantity $\rho_{e}^{v}$ qg mod is defined in eq. 23 , here with $v=0.975$, the same value as used in the subsequent figures $7-9$. 
EPJ Web of Conferences

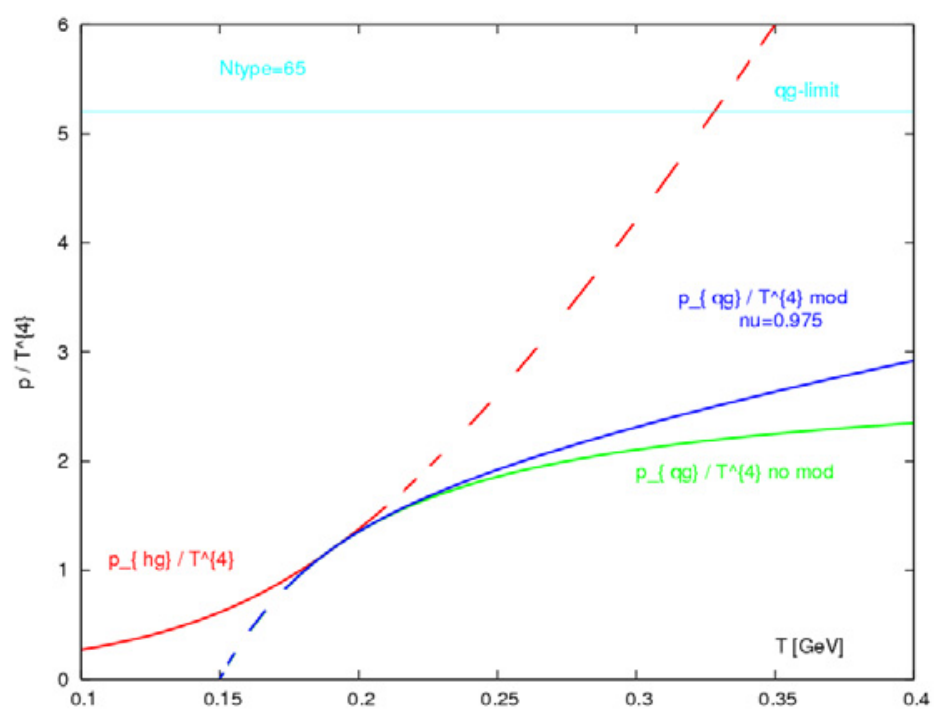

Figure 7. The enumeration in subsection 2.3 with respect to Figure 7 is repeated below.

It shows the (dominantly) third order nature of the transition under the same conditions as underlying figure 6 for the quantity $p / T^{4}$, piecewise described by $p_{e h g(-65)} / T^{4}$ for $T \leq T_{c}$ from the HRG side and $p_{\text {qg no mod (mod) }} / T^{4}$ for $T \geq T_{c}$ from the quark-gluon side.

The three curves are represented with dashed lines outside their range of validity. The quantity $p_{q g \text { mod }}^{v}$ is defined in eq. 23, here with $v=0.975$, as used in the subsequent figures $8-9$.

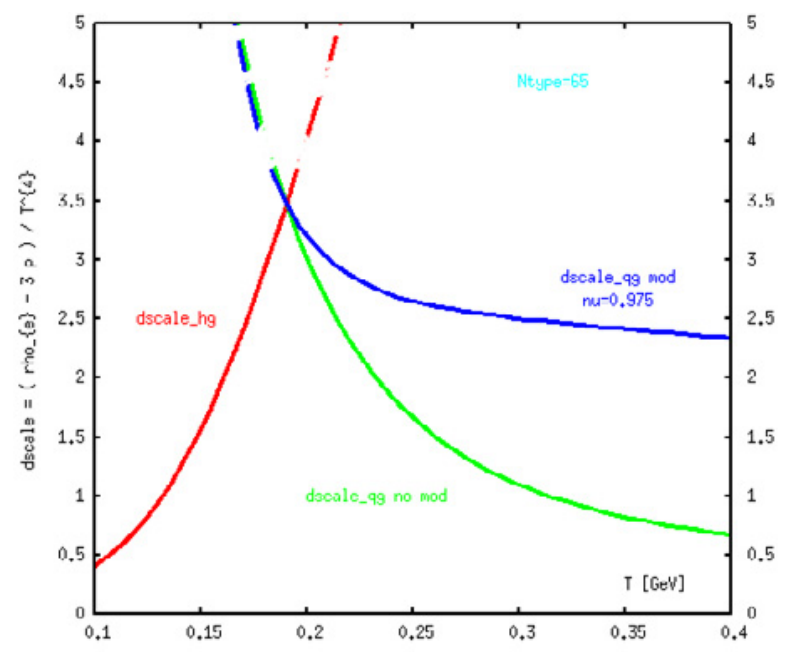

Figure 8. The 'indian tent'. The enumeration in subsection 2.3 with respect to Figure 8 is repeated below.

It shows the (dominantly) second order nature of the transition under the same conditions as figures 6 and 7 for the quantity dscale $=\left(\varrho_{e}-3 p\right) / T^{4}$, displaying the form of an 'indian 
tent'. The transition is piecewise described by dscale ${ }_{h g}$ for $T \leq T_{c}$ from the HRG side and dscale $_{q g}$ no mod (mod) for $T \geq T_{c}$ from the quark-gluon side.

The three curves are represented with dashed lines outside their range of validity. The quantity dscale $_{q q \text { mod }}^{v}$ is obtained from eq. 23, here with $v=0.975$, as used in the subsequent figure 9 .

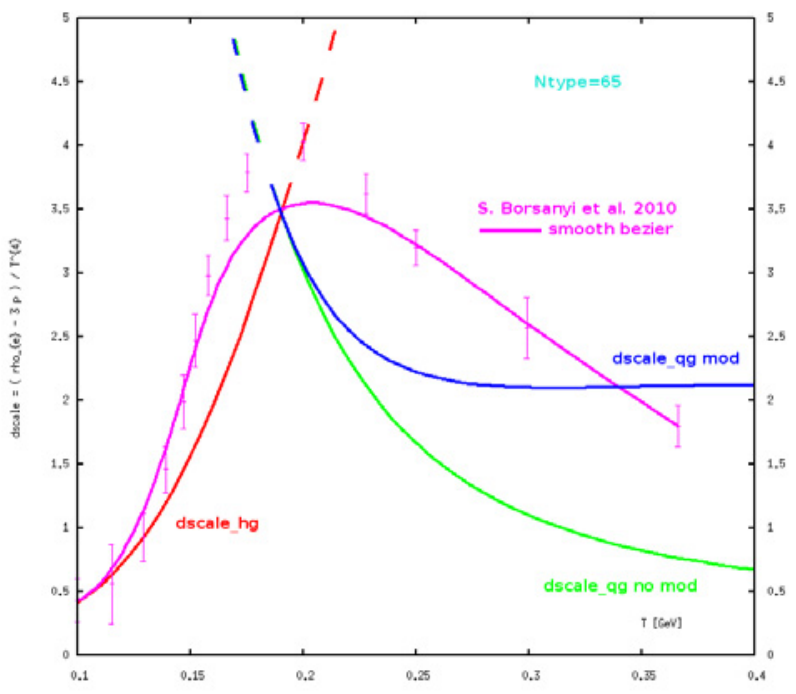

Figure 9. The 'indian tent' as compared with the lattice QCD calculation of dscale, also called the trace anomaly, by S. Borsanyi et al. [7-2010]. The enumeration in subsection 2.3 with respect to Figure 9 is repeated below.

It shows the quantities (dscale) forming the 'indian tent' identical to figure 5. The same quantity as obtained in ref. [7-2010] from lattice simulation of QCD under the same thermal conditions is also plotted for comparison.

The three curves forming the 'indian tent' are represented with dashed lines outside their range of validity. The quantity dscale ${ }_{q g \text { mod }}^{v}$ is obtained from eq. 23 , here with $v=0.975$, the same as in figures $6-8$.

It shows the first order nature of the transition of the square of the velocity of sound, under the same conditions as for figures 6 - 9, piecewise described by the quantities $v_{h g}^{2}$ for $T \leq T_{c}$ from the HRG side and $v_{q g \text { no mod }}^{2}$ for $T \geq T_{c}$ from the quark-gluon side. Only the unmodified setting is used for $v_{g q}^{2}$.

The two curves for $v_{h g}^{2} v_{g q}^{2}$ are represented with dashed lines outside their range of validity.

\section{Outlook}

The theoretical considerations concerning the phase structure of QCD for vanishing chemical potentials lead us to the illustration in principle of thermodynamic energy density and pressure in the vicinity of the critical temperature $T_{c} \sim 200 \mathrm{Mev}$, reflecting the resolution of the infrared instability of QCD through Bose condensation of gauge invariantly paired gauge bosons, a basic form of such condensation represented by the bosonic version of Bogoliubov transformations [H3-1947]. 


\section{EPJ Web of Conferences}

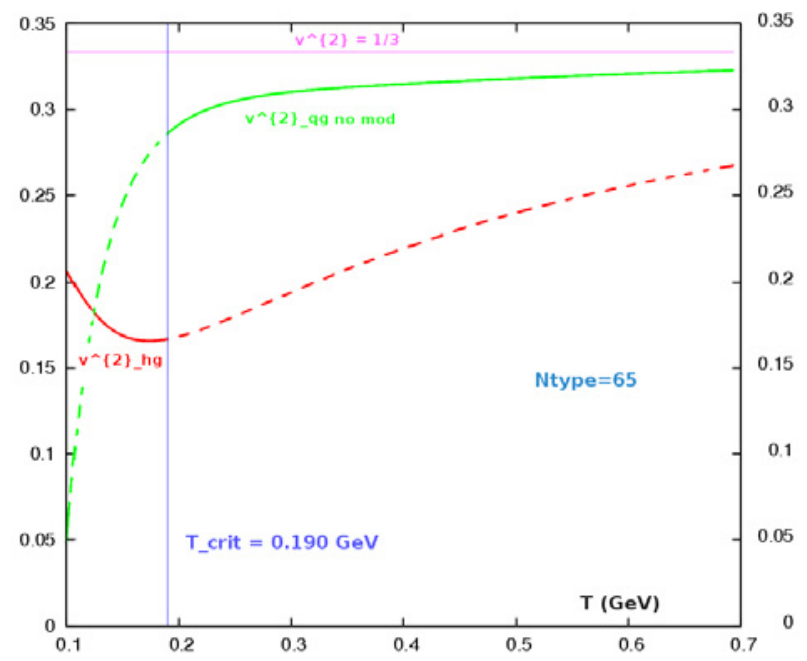

Figure 10. To Fig. 10 : The square of the velocity of sound. The enumeration in subsection 2.3 with respect to Figure 10 is repeated below.

A comparison with the strong coupling regime observed in central Au Au collisions at $200 \mathrm{GeV}$ at RHIC [13-2005, 14-2005] and the continuation of measurements of $\mathrm{p} p$ as well as $\mathrm{Pb} \mathrm{Pb}$ collisions by the ALICE collaboration at LHC combined with heavy ion collisions at lower center of mass energy offers the possibility to deduce thermodynamic base variables of QCD, albeit indirectly, complementing a longstanding effort, experimental and theoretical, in this direction. The theoretical description in lattice simulations of QCD offers a direct access to all thermal equilibrium states.

The hypotheses adopted in the paper on which this report is based predict in the neighbourhood of vanishing chemical potentials a phase transition, of (essentially) second order with respect to energy density, as illustrated in figures 4 - 10.

The goal remains to actually prove or disprove the hypotheses and their consequences discussed here, wide open to, ideally, experimental and theoretical investigation and interpretation.

\section{References}

[1-2010] S. Kabana and P. Minkowski, 'On the thermal phase structure of QCD at vanishing chemical potentials', Jan 2010, 7 pp., CERN-PH-TH-2010-002, Int.J.Mod.Phys. A26 (2011) 3035-3050, arXiv:1001.0707 [hep-ph].

[2-1993] W. H. Zurek, 'Cosmic strings in laboratory superfluids and remnants of other phase transitions', Acta Physica Polonica B7 (1993) 1301,

URL $\rightarrow$ http://th-www.if.uj.edu.pl/acta/vol24/pdf/v24p1301.pdf.

[3-2011] D. Vollhardt, Center for Electronic Correlations and Magnetism, University of Augsburg, 'Superfluid Phases of Helium-3 : From very low Temperatures to the Big Bang', Superfluid_3He_Prague_2011-06-08_homepage.pdf.

[4-2001] Bardeen, Cooper and Schrieffer, Chapter 10 : Superconductivity, www.teknik.uu.se/ftf/education/ftf2/SC_BCS_theory.pdf. 
[5-1973] J.M. Kosterlitz and D.J. Thouless, 'Ordering, metastability and phase transitions in twodimensional systems', J.Phys.C6 (1973) 1181-1203.

[6-2001] S. Kabana and P. Minkowski, 'Mapping out the QCD phase transition in multiparticle production', BUTP-2000-26, BUHE-00-09, Oct 2000, 53pp., New J.Phys.3 (2001) 4, hep$\mathrm{ph} / 0010247$.

[7-2010] Szabolcs Borsanyi, Gergely Endrodi, Zoltan Fodor, Antal Jakovac, Sandor D. Katz, Stefan Krieg, Claudia Ratti, Kalman K. Szabo, 'The QCD equation of state with dynamical quarks', WUB/10-12, MIT-CTP 4168, JHEP11(2010)077, arXiv:1007.2580v2 [hep-lat].

[8-2009] The MILC Collaboration (A. Bazavov et al.), 'Results from the MILC collaboration's SU(3) chiral perturbation theory analysis', Oct 2009. 7pp., to appear in the proceedings of 27th International Symposium on Lattice Field Theory (Lattice 2009), Beijing, China, 25-31 July 2009, PoS LAT2009 (2009) 079, arXiv:0910.3618 [hep-lat].

[9-1979] M. A. Shifman, A. I. Vainshtein and V. I. Zakharov, 'QCD and resonance physics. Theoretical Foundations.', Nucl. Phys. B147 (1979) 385, 448.

[10-1998] S. Narison, 'Masses, decays and mixings of gluonia in QCD,' PM-96-37, Dec 1996. 32pp., Nucl. Phys. B509 (1998) 312, hep-ph/9612457i.

[11-2007] A. Ipp, K. Kajantie, A. Rebhan and A. Vuorinen, 'Unified description of deconfined QCD equation of state', J. Phys. G: Nucl. Part. Phys. 34 (2007) 631 and references cited therein.

[12-1988] P. Minkowski, 'Color Superconductivity In QCd And The Associated Phase Transition', Bern University preprint BUTP-88-22, Sep 1988. 52pp., Czech.Hadron Symp.1988:279.

[13-2005] STAR coll., Nucl. Phys. A 757 (2005) 102,

Brahms coll., Nucl. Phys. A 757 (2005) 1,

Phenix coll., Nucl. Phys. A 757 (2005) 184,

Phobos coll., Nucl. Phys. A 757 (2005) 28.

[14-2005] E. V. Shuryak, Nucl. Phys. A 750, 64 (2005), arXiv:hep-ph/0405066.

[H1-1937] P. L. Kapitsa, Nature 141 (1937) 74, JETP 11 (1941), 1 and 581.

[H2-1941] L. D. Landau, JETP 11 (1941) 592 and L. D. Landau and E. M. Lifshitz, 'Statistical Mechanics', Pergamon Press, New York 1958.

[H3-1947] N. N. Bogoliubov, J. Phys. (USSR) 11 (1947) 23.

[18] L. N. Cooper, 'Bound Electron Pairs in a Degenerate Fermi Gas', Phys. Rev 104 (1956) 1189 - 1190,

J. Bardeen, L. N. Cooper, and J. R. Schrieffer, 'Microscopic Theory of Superconductivity',

Phys. Rev. 106 (1957) 162 - 164,

J. Bardeen, L. N. Cooper, and J. R. Schrieffer, 'Theory of Superconductivity', Phys. Rev. 108 (1957) 1175.

[19] L. D. Landau, 'The theory of a Fermi liquid', JETP 3 (1957) 920 (english translation), original : JETP 30 (1956) 1058 ;

'Oscillations in a Fermi liquid', JETP 5 (1957) 101 (english translation).

[H6-2002] J.M. Vogels,K. Xu,C. Raman,J.R. Abo-Shaeer and W. Ketterle, 'Experimental observation of the Bogolyubov transformation for a Bose-Einstein condensed gas',

Phys.Rev.Lett.88 (2002) 060402, cond-mat/0109205, and references cited therein. 